\title{
Passivity based control of hydraulic linear arms using natural Casimir functions
}

\author{
Satoru Sakai \\ Chiba University \\ Japan
}

\section{INTRODUCTION}

This chapter discusses a modeling and passivity based control of hydraulic arms which are robotic, that is, have asymmetric cylinders. Hydraulic arms are very important components in field robotics, such as construction, agriculture, rescue, demining robotics and so on since hydraulic arms are superior to electric arms with respect to the power to weight ratio and also can keep joint forces even when the energy source (the hydraulic pump) does not work.

In many cases of electric arms, the driving system (or the actuator dynamics) is simple and almost static, for example, the input torque (or velocity) is just proportional to the control input. On the other hand, in many cases of hydraulic arms, the driving system is complex and consists of compressible fluid systems, that is, nonlinear dynamical systems with unknown (or hard-to-be identified) parameters. To solve these problems, this chapter gives some results about modeling and control of hydraulic arms by applying and developing port-Hamiltonian systems and control theory.

Port-Hamiltonian systems van der Schaft (2000) are generalization of Hamiltonian systems in classical mechanics but can model many systems such as electro-mechanical systems, mechanical systems with nonholonomic constraints Maschke \& van der Schaft (1994), distributed systems. The first important property of port-Hamiltonian systems is that the interconnection of port-Hamiltonian systems gives again another port-Hamiltonian system. That is, it is easy to treat more complex systems consisting of these finite systems and the infinite systems such as the flexible beams Macchelli \& Melchiorri (2005). The second important property of port-Hamiltonian systems is passivity and some passivity based control methods, originally from the chapter Takegaki \& Arimoto (1981), were developed, such as, EnergyCasimir methods van der Schaft (2000), the generalized canonical transformations Fujimoto \& Sugie (2001), IDA-PBC Ortega \& Garcia-Canseco (2004) and IPC approach Sakai \& Stramigioli (2007); Stramigioli et al. (1998) and so on. These methods can give nonlinear robust controllers, and not only stabilization, but also tracking and dynamic output feedback stabilization Sakai \& Fujimoto (2005) are achieved already.

For hydraulic arms, some nonliner robust (or adaptive) controllers were already proposed Bonchis et al. (2001); Mazenc \& Richard (n.d.); Yao et al. (2000); Zhu \& Piedboeuf (n.d.). However, in these approaches, the closed-loop systems are not port-Hamiltonian systems any more, even when the controlled systems can be described as the port-Hamiltonian systems. 
That is, it is difficult to treat and extend the results in these approaches to more complex systems such as the high-degree of freedom (hydraulic) mechanisms and the dynamic energy sources.

Recently, modeling and control of several fluid systems are discussed in port-Hamiltonian form. For example, Ramkrishna et al. discuss infinite dimensional canal systems in three dimensional space Ramkrishna \& van. der. Schaft (2006) . These fluid systems have free-surface and are incompressible, that is, differ from compressible fluid systems in hydraulic arms. Riccardo et al. discuss the modeling of hydraulic arms and show some experimental results Riccardo et al. (2006). Gernot et al. discuss the control of hydraulic arms Gernot \& Schlacher (2005). However, these approaches are based on the standard procedure using only the first or the second properties above. Apart from these approaches, we discuss the modeling and control of hydraulic arms based on a new (the third important) structural property, that is, not only passivity but also Casimir functions are used in the modeling and control as a new structural property. In our approach, the bulk modulus is not identified at all.

This chapter gives a new passivity based control of hydraulic arms based on a new model using natural Casimir functions. In Section II, we refer port-Hamiltonian systems and their properties. In Section III, we propose two stabilization methods, a new dynamic asymptotic stabilization method and a new partial stabilization method. In Section IV, we give a new model of hydraulic arms using Casimir functions. At the same time, a very fundamental state of hydraulic arms is discovered. In Section V, the proposed two stabilization methods are applied to the new model of hydraulic arms and a new passivity based control of hydraulic arms are proposed. In Section VI, the validity of our methods are confirmed by numerical simulation and finally we conclude this chapter in Section VII.

In this chapter, $I_{n}$ is $n \times n$ identity matrix, $\mathbb{R}^{m \times n}$ is the space of the $m$ rows and $n$ columns real matrix. Class- $\mathcal{K}$ functions are strictly increasing functions which have the origin Khalil (1996).

\section{Port-Hamiltonian systems}

\subsection{Port-Hamiltonian systems}

A port-Hamiltonian system with a Hamiltonian $H(x) \in \mathbb{R}$ is a system described by

$$
\left\{\begin{aligned}
\dot{x} & =J(x) \frac{\partial H(x)}{\partial x}^{\mathrm{T}}+g(x) u \\
y & =g(x)^{\mathrm{T}} \frac{\partial H(x)}{\partial x}^{\mathrm{T}}
\end{aligned}\right.
$$

with $u, y \in \mathbb{R}^{m}, x \in \mathbb{R}^{n}$ and a skew symmetric matrix $J(x)$, i.e. $-J(x)=J(x)^{\mathrm{T}}$ holds ${ }^{1}$. Hamiltonian $H$ is bounded from below. The following first property is known Maschke \& van der Schaft (1992).

Lemma 1 van der Schaft (2000) Consider the port-Hamiltonian system (1). Suppose the Hamiltonian $H(x)$ satisfies $H(x) \geq H(0)=0$. Then the input-output mapping $u \mapsto y$ of the system is passive with respect to the storage function $H$, and the feedback

$$
u=-D(x) y
$$

\footnotetext{
${ }^{1} J$ was replaced by a negative semidefinite matrix in order to describe dissipative elements van der Schaft (2000). The results in this chapter can be directly applicable to this modification, Nevertheless we concentrate on a skew-symmetric matrix $J$ in this chapter for simplicity.
} 
with a matrix $D(x) \geq \varepsilon I>0 \in \mathbb{R}^{m \times m}$ renders $(u, y) \rightarrow 0$. Furthermore if $H(x)$ is positive definite and if the system is zero-state detectable, then the feedback (2) renders the origin asymptotically stable. The zero-state detectability and the positive definiteness of the Hamiltonian assumed in Lemma 1 do not always hold for general port-Hamiltonian systems. In such a case, the generalized canonical transformation is useful.

\subsection{Casimir functions}

One of other properties of port-Hamiltonian systems are the existence of Casimir functions. Casimir functions (with respect to $J$ ) are defined as solutions of the following PDE,

$$
\frac{\partial C(x)}{\partial x} J(x) \equiv 0
$$

Not only Hamiltonian, but also Casimir functions are the special first integrals, that is,

$$
\dot{C} \equiv 0
$$

holds for any Hamiltonian $H(x)$ when $u=0$. Unlike Hamiltonian, Casimir functions are not bounded from below nor upper in general and do not alway exist for port-Hamiltonian systems (but exist in some important cases in robotics).

Note that we do not treat Casimir functions for closed-loop systems but treat "Casimir functions for controlled systems (plants)". The former Casimir functions are artificial and discussed in controller design phase such as the Energy-Casimir method van der Schaft (2000). The latter Casimir functions are discussed in (at least) modeling phase and referred to as "natural Casimir functions" to avoid confusions in this chapter.

\section{Dynamic and partial stabilization for port-Hamiltonian systems}

\subsection{Dynamic asymptotic stabilization for port-Hamiltonian systems}

In this subsection, we give a dynamic stabilization method for port-Hamiltonian systems. As we refereed in the previous section, port-Hamiltonian systems can be stabilized by the static stabilizers. However, a dynamic stabilizers are also useful and will be applied to hydraulic arms later.

Theorem 1 Consider the following (mechanical) port-Hamiltonian systems

$$
\Sigma_{m}^{p H}:\left\{\begin{array}{l}
{\left[\begin{array}{c}
\dot{q} \\
\dot{p}
\end{array}\right]=\left[\begin{array}{cc}
0 & I \\
-I & 0
\end{array}\right]\left[\begin{array}{c}
\frac{\partial H_{m} T}{\partial q} \\
\frac{\partial H_{m}}{\partial p}
\end{array}\right]+\left[\begin{array}{l}
0 \\
G
\end{array}\right] u} \\
y=G^{T} \frac{\partial H_{m}}{\partial p}{ }^{T}
\end{array}\right.
$$

where $q, p \in \mathbb{R}^{n}$ are the (generalized) position and momentum, $G$ is nonsingular matrix and the Hamiltonian $H_{m}=(1 / 2)\left(p^{T} M(q)^{-1} p\right)+U(q)$ with $M=M^{T}>0$ and $U(q) \geq U(0)=0$. Then, the following dynamic controller

$$
\Sigma_{d d}:\left\{\begin{array}{l}
\dot{r}=P(x, r)^{T} G^{-T} y-D(x, r){\frac{\partial H_{r}}{\partial r}}^{T} \\
u=-P(x, r){\frac{\partial H_{r}}{\partial r}}^{T}
\end{array}\right.
$$


makes the set $\Omega_{0}=\{(q, p) \mid y=u=0\}$ asymptotically stable, where $x=(q, p)^{T} \in \mathbb{R}^{2 n}, r \in \mathbb{R}^{r}$ $H_{r}=(1 / 2) r^{T} R(r) r$ with $R=R^{T}>0, P^{T} \in \mathbb{R}^{r \times m}$ and $D=D^{T}>0$.

Proof of Theorem 1

From Equations (5) and (6), the closed-loop system is

$$
\Sigma_{c l}^{p H}:\left\{\left[\begin{array}{l}
\dot{q} \\
\dot{p} \\
\dot{r}
\end{array}\right]=\left[\begin{array}{ccc}
0 & I & 0 \\
-I & 0 & -P(x, r) \\
0 & P(x, r)^{T} & -D(x, r)
\end{array}\right]\left[\begin{array}{l}
\left.\frac{\partial\left(H_{m}+H_{r}\right)}{\partial q}\right)^{T} \\
\frac{\partial\left(H_{m}+H_{r}\right)^{T}}{\partial p} \\
\left.\frac{\partial\left(H_{m}+H_{r}\right)}{\partial r}\right)^{T}
\end{array}\right]\right.
$$

and (zero-input) port-Hamiltonian systems with a new Hamiltonian $H_{m}+H_{r}$ and a dissipation. Since the time derivative of $H+H_{r}$ (along the trajectory) is

$$
\dot{H}+\dot{H}_{r}=-\frac{\partial H_{r}}{\partial r} D{\frac{\partial H_{r}}{\partial r}}^{T} \leq 0
$$

and $H+H_{r}$ is bounded from below,

$$
{\frac{\partial H_{r}}{\partial r}}^{T} \rightarrow 0
$$

as $t \rightarrow \infty$, that is, the set $\{u=0\}$ is asymptotically stable from Equation (6).

At the same time, Equation (9) implies $r \rightarrow 0$, and thus $\dot{r} \rightarrow 0$ because $H_{r}$ is a class- $\mathcal{K}$ function with respect to $\|r\|$. Since we have Equation (6) and

$$
P(x)^{T} \frac{\partial\left(H_{m}+H_{r}\right)^{T}}{\partial p}=0 \Rightarrow \frac{\partial H_{m}{ }^{T}}{\partial p}=0
$$

due to the condition $m \geq r$, the set $\{y=0\}$ is asymptotically stable from Equation (5). In all, the set $\{y=u=0\}$ is asymptotically stable. (Q.E.D.)

Note that the proposed dynamic asymptotic stabilization is a generalization of our result Sakai \& Fujimoto (2005). The origin of the closed-loop system (7) is asymptotically stable if and only if the origin of a closed-loop system from Equations (5) and (2) is asymptotically stable.

It is easy for readers to extend the mechanical port-Hamiltonian system $\Sigma_{m}^{p H}$ in Theorem 1 to more general port-Hamiltonian systems even though Theorem 1 will be applied directly later.

\subsection{Partial stabilization for port-Hamiltonian systems with Casimir function}

Advantages of port-Hamiltonian systems are from their structural properties, such as passivity, which do not exist in general nonlinear systems. In this subsection, we focus a new structural property of a special port-Hamiltonian systems, that is, port-Hamiltonian systems with Casimir functions. As we referred in Section II, Casimir functions do not exist in general port-Hamiltonian systems.

One of the new advantages of this special port-Hamitonian systems are presented in the following theorem.

Theorem 2 Consider the following port-Hamiltonian systems with Casimir functions $C(x)$

$$
\Sigma_{c}^{p H}:\left\{\begin{array}{c}
{\left[\begin{array}{c}
\dot{q} \\
\dot{p}
\end{array}\right]=J(q, p)\left[\begin{array}{c}
\frac{\partial H}{\partial q}^{T} \\
\frac{\partial H}{\partial p}^{T}
\end{array}\right]+\left[\begin{array}{l}
0 \\
G
\end{array}\right] u} \\
y=G^{T} \frac{\partial H}{\partial p}^{T}
\end{array}\right.
$$


where $q, p \in \mathbb{R}^{n}$ are the (generalized) position and momentum, $G$ is nonsingular matrix and the Hamiltonian $H \geq H(0)=0$. Suppose there exists a coordinate transformation $x \mapsto \phi(x)=\left(x_{r}, C\right)^{T}$ such that

$$
H(\phi)=H_{r}\left(x_{r}\right)+H_{c}(C),
$$

where $u, y \in \mathbb{R}^{m}, x_{r} \in \mathbb{R}^{r}, r \leq \operatorname{rank}(J)$ and $H_{r}$ is bounded from below.

Then the feedback

$$
u=-D(x) y_{r}
$$

with $D=D^{T}>0$ and

$$
y_{r}=\left[\begin{array}{ll}
0 & G^{T}
\end{array}\right]{\frac{\partial \phi^{T}}{\partial x}}^{T}\left[\begin{array}{c}
I_{r} \\
0
\end{array}\right] \frac{\partial H_{r}}{\partial x_{r}}
$$

makes the set $\left\{y_{r}=0\right\}$ asymptotically stable.

\section{Proof of Theorem 2}

From the existence of Casimir functions $C(x)$, there exists a coordinate transformation which converts the system (11) into

$$
\left\{\begin{array}{c}
{\left[\begin{array}{c}
\dot{x}_{r} \\
\dot{C}
\end{array}\right]=\left[\begin{array}{cc}
J_{r}\left(x_{r}, C\right) & 0 \\
0 & 0
\end{array}\right]\left[\begin{array}{c}
{\frac{\partial H\left(x_{r}, C\right)}{\partial x_{r}}}^{T} \\
{\frac{\partial H\left(x_{r}, C\right)}{\partial C}}^{T}
\end{array}\right]+\frac{\partial \phi}{\partial x}\left[\begin{array}{l}
0 \\
G
\end{array}\right] u} \\
y=\left[0 G^{T}\right] \frac{\partial \phi}{\partial x}^{T} \frac{\partial H}{\partial \phi}^{T}
\end{array}\right.
$$

with the skew-symmetrix matrix $J_{r}$.

The time derivative (along the trajectory) of the function $H_{r}$ is given as

$$
\begin{aligned}
& \dot{H}_{r}=\frac{\partial H_{r}}{\partial x_{r}} \dot{x}_{r}+\frac{\partial H_{r}}{\partial C} \dot{C}
\end{aligned}
$$

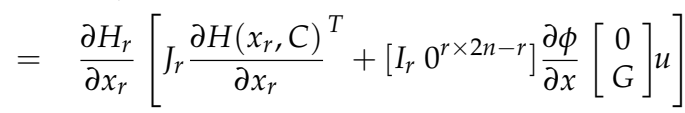

$$
\begin{aligned}
& =\frac{\partial H_{r}}{\partial x_{r}}\left[J_{r} \frac{\partial H_{r}{ }^{T}}{\partial x_{r}}+\left[\begin{array}{lll}
I_{r} & 0
\end{array}\right] \frac{\partial \phi}{\partial x}\left[\begin{array}{l}
0 \\
G
\end{array}\right] u\right] \\
& =\frac{\partial H_{r}}{\partial x_{r}}\left[I_{r} 0\right] \frac{\partial \phi}{\partial x}\left[\begin{array}{l}
0 \\
G
\end{array}\right] u
\end{aligned}
$$

due to the special Hamiltonian structure $H=H_{r}\left(x_{r}\right)+H_{c}(C)$.

This means that the system with input $u$ and output $y_{r}$ is passive (lossless) with respect to the storage function $H_{r}$, that is,

$$
\dot{H}_{r}=y_{r}^{T} u
$$

holds. Finally the controller (13) makes the set $\left\{y_{r}=0\right\}$ asymptotically stable since

$$
\dot{H}_{r}=-y_{r}^{T} D y_{r} \leq 0
$$

and $H_{r}$ is bounded from below. (Q.E.D.) 
Note that the above output $y_{r}$ is different from the usual output $y$ of port-Hamiltonian function and a new output based on the structural properties of special port-Hamiltonian systems, that is, port-Hamiltonian systems with Casimir functions. Furthermore, not all states, but only the partial state $x_{r}$ is stabilized in Theorem 2 because $H_{r}$ is the function of $x_{r}$.

By combination of the above two proposed stabilization methods, that is, the dynamic stabilization method and the partial stabilization method, robotic hydraulic arms will be controlled later based on the modeling in the next section.

\section{Modeling of hydraulic arms using natural Casimir functions}

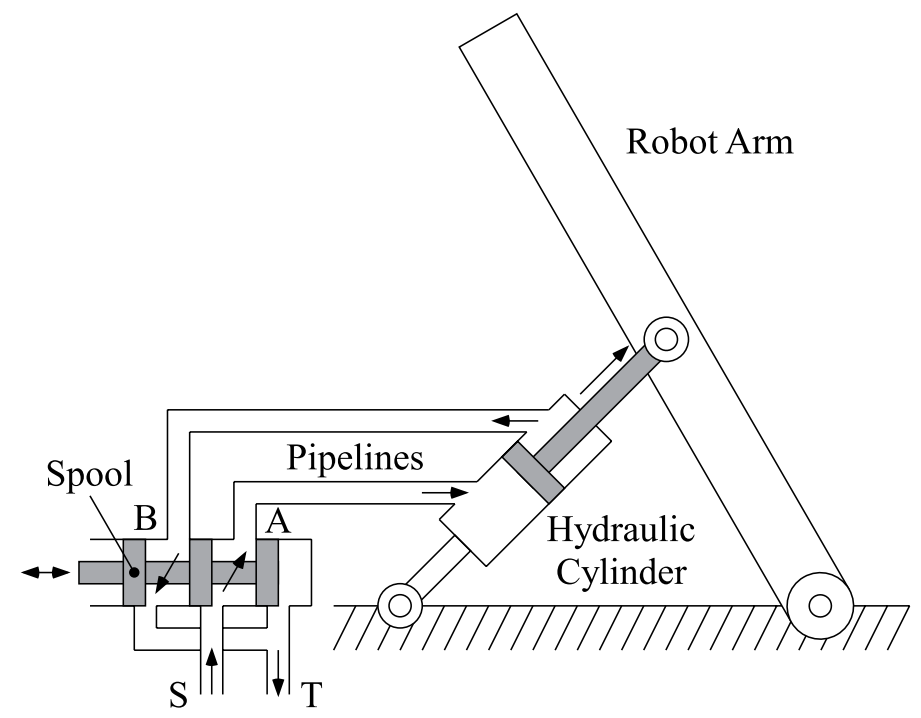

Fig. 1. Hydraulic arms

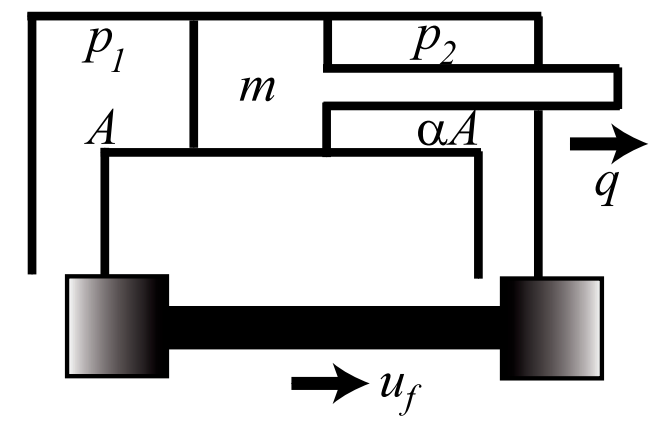

Fig. 2. The equivalent model of hydraulic arms

In this section, modeling of robotic hydraulic arm (with asymmetric cylinders as shown in Fig. 1) is discussed in port-Hamiltonian framework. This section discusses an empirical model 
in Merrit (1967) and Jelali \& Kroll (2002), which is equivalent to the model in Fig. 2. The spool in Fig. 2 has only two slots while the original one in Fig. 1 has three slots. The empirical model is not infinite dimensional but finite dimensional model, and takes input not as the driving force of the spool, but as the spool displacement. This finite dimensional model with spool displacement input is based some assumptions but already used in many theoretical and experimental works Bonchis et al. (2001); Mazenc \& Richard (n.d.); Yao et al. (2000); Zhu \& Piedboeuf (n.d.).

\subsection{Generalized continuous law}

A continuous law (in integral form) for compressible fluid is

$$
\frac{V}{E} \dot{p}_{i}=-\dot{V}+Q_{i}^{i n}-Q_{i}^{\text {out }}
$$

where $p_{i}$ is the pressure of chamber $i, Q_{i}^{\text {in }}$ is the flow into chamber $i, Q_{i}^{\text {out }}$ is the flow from chamber $i, E$ is the bulk modulus and $V$ is the fluid volume. See Jelali \& Kroll (2002) for this modeling assumptions in detail.

As the port-Hamiltonian systems are generalization of classical Hamiltonian system (the energy conservation law), the following system is given as a generalization of the continuous law (19)

where

$$
\Sigma_{f}:\left\{\begin{array}{c}
{\left[\begin{array}{c}
\dot{x}_{f 1} \\
\dot{x}_{f 2}
\end{array}\right]=\left[\begin{array}{c}
-A \\
\alpha A
\end{array}\right] u_{o}+\left[\begin{array}{l}
g_{p 1} \\
g_{p 2}
\end{array}\right] u_{f}} \\
y_{f}=\left[\begin{array}{ll}
-A \alpha]{\frac{H_{f}}{\partial x_{f}}}^{T}
\end{array}\right.
\end{array}\right.
$$

$$
H_{f}=\frac{E}{2 V}\left(x_{f 1}^{2}+x_{f 2}^{2}\right)
$$

$x_{f}=\left(x_{f 1}, x_{f 2}\right)^{T}, x_{f i}=(V / E) p_{i}, u_{o}$ is the cylinder velocity and $g_{p i}=g_{p i}\left(x_{f i}\right)$ are the flow velocity from Bernoulli's equation (omitted from its uniqueness), $u_{f}$ is the spool input displacement, $A$ and $\alpha A$ are the square in the chambers. Since the cylinder is asymmetric, $0<\alpha<1$ holds. The state is not pressure and different from that in the previous model Gernot \& Schlacher (2005).

Now the mechanical system $\Sigma_{m}^{p H}$ and the fluid system $\Sigma_{f}$ are interconnected by the following

$$
\left\{\begin{array}{lll}
u_{0} & = & y \\
u & = & -y_{f}
\end{array}\right.
$$

because $u_{o}$ is the cylinder velocity and $y_{f}$ is the driving force of the cylinder. Then the interconnected system is given as

$$
\Sigma_{f m}^{p H}:\left\{\begin{array}{c}
{\left[\begin{array}{c}
\dot{q} \\
\dot{p} \\
\dot{x}_{f 1} \\
\dot{x}_{f 2}
\end{array}\right]=\left[\begin{array}{cccc}
0 & I & 0 & 0 \\
-I & 0 & G A & -\alpha G A \\
0 & -A G^{T} & 0 & 0 \\
0 & \alpha A G^{T} & 0 & 0
\end{array}\right]\left[\begin{array}{c}
\frac{\partial H_{f m} T}{\partial q^{T}} \\
\frac{\partial H_{f m} T}{\partial p} \\
\frac{\partial H_{f m} T}{\partial x_{f 1}}{ }^{T} H_{f m} \\
\frac{\partial x_{f 2}}{2}
\end{array}\right]+\left[\begin{array}{c}
0 \\
G_{f}
\end{array}\right] u_{f}} \\
y_{f 1}=G_{f}^{T} \frac{\partial H_{f m}{ }^{T}}{\partial x_{f}}
\end{array}\right.
$$


where $H_{f m}=H_{f}+H_{m}$ and $G_{f}=\left[\begin{array}{ll}g_{p 1} & g_{p 2}\end{array}\right]^{T} . \Sigma_{f m}^{p H}$ is easily confirmed to be port-Hamiltonian systems since $J$ matrix part is again skew symmetric.

It is interesting that the interconnected system of the fluid system $\Sigma_{f}$ and the mechanical system $\Sigma_{m}^{p H}$ is again a port-Hamiltonian system even though the fluid system $\Sigma_{f}$ is not portHamiltonian system. By the generalized continuous law, we can take not only the energy conservation but also the mass conservation into account simultaneously. This situation is different from that in fluid mechanics where only the energy conservation is taken in NavierStokes equations and only the mass conservation is taken in the continuous law.

\subsection{Casimir functions}

In this section, we give the most important result on the modeling of hydraulic arms.

Lemma 2 Consider the fluid-mechanical port-Hamiltonian systems $\Sigma_{f m}$. Then there exists a Casimir function

$$
C_{f}=\frac{1}{\sqrt{1+\alpha^{2}}}\left(\alpha x_{f 1}+x_{f 2}\right)
$$

\section{Proof of Lemma 2.}

By a direct calculation, it is confirmed that $C_{f}$ satisfies the PDE (3), that is, $\dot{C}_{f} \equiv 0$ holds for any Hamiltonian $H_{f m}$ at zero-input. (Q.E.D.)

Theorem 3 Consider the fluid-mechanical port-Hamiltonian systems $\Sigma_{f m}$. Then there exists a coordinate transformation $\phi$ such that the transformed systems satisfy the condition (12).

Proof of Theorem 3.

Consider the following coordinate transformation,

$$
\left[\begin{array}{c}
q \\
p \\
x_{f r} \\
C_{f}
\end{array}\right]=\left[\begin{array}{ccc}
I_{2 n} & 0 & 0 \\
0 & \frac{1}{\sqrt{1+\alpha^{2}}} & \frac{-\alpha}{\sqrt{1+\alpha^{2}}} \\
0 & \frac{\alpha}{\sqrt{1+\alpha^{2}}} & \frac{-1}{\sqrt{1+\alpha^{2}}}
\end{array}\right]\left[\begin{array}{c}
q \\
p \\
x_{f 1} \\
x_{f 2}
\end{array}\right]
$$

where $C_{f}$ is already given in Lemma 2. It is calculated that the system (23) are transformed to a new port-Hamiltonian systems which satisfies the conditions (12) because we have $H=$ $H_{r}+(1 / 2) C_{f}^{T} C_{f}$ where $H_{r}$ is given in the following $J_{r}$-part dynamics (a reduced dynamics) in (15)

$$
\left\{\begin{aligned}
{\left[\begin{array}{c}
\dot{q} \\
\dot{p} \\
x_{f r}^{\cdot}
\end{array}\right]=\left[\begin{array}{ccc}
0 & I & 0 \\
-I & 0 & \sqrt{1+\alpha^{2}} A \\
0 & -\sqrt{1+\alpha^{2}} A & 0
\end{array}\right]\left[\begin{array}{c}
\frac{\partial H_{r}}{\partial q} \\
\frac{\partial H_{r}}{\partial p} \\
\frac{\partial H_{r}}{\partial x_{f r}}
\end{array}\right]+\left[\begin{array}{c}
0 \\
g_{r}
\end{array}\right] u_{f} } \\
y_{r}=g_{r}^{T} \frac{\partial_{H_{r}}{ }^{T}}{\partial x_{f r}}
\end{aligned}\right.
$$

where

$$
H_{r}=H_{m}+\frac{E}{2 V} x_{f r}^{2}
$$

and $g_{r}$ is omitted because of its uniqueness. (Q.E.D.) 


\section{Control of hydraulic arms using natural Casimir functions}

In this section, we give a new stabilization method based on the previous results in this chapter. The proposed controller in this section can stabilize only mechanical part, even if the parameters of the fluid systems, the bulk modules $E$, is unknown.

Lemma 3 Consider the fluid-mechanical systems $\Sigma_{f m}^{p h}$ and suppose that $U(q)$ is the positive definite function. Then the feedback

$$
u_{f}=-D(x) g_{r}^{T}{\frac{\partial H_{r}}{\partial x_{f r}}}^{T}
$$

with a matrix $D(x) \geq \varepsilon I>0 \in \mathbb{R}^{m \times m}$ makes the set $\{(q, p)=0\}$ asymptotically stable.

Proof of Lemma 3.

First, from Theorem 2 and Theorem 3, the set

$$
\left\{y_{r}=g_{r}^{T}{\frac{\partial H_{r}}{\partial x_{f r}}}^{T}=0\right\}
$$

is asymptotically stable. Second, since the closed-loop system of (26) and (28) is equivalent to the system (7) in Theorem 1, the feedback (28) makes the set

$$
\left\{y=\frac{\partial H_{m}{ }^{T}}{\partial p}=0\right\}
$$

asymptotically stable. This implies that the set $\{(q, p)=0\}$ is asymptotically stabilized because the zero-state detectability holds due to the positive definiteness of $U(q)$. (Q.E.D.)

Note that the above stabilization is achieved without the exact value of $E$, that is, the parameter identification is not required since all inequalities hold for any $E>0$. Furthermore, the closed-loop system keeps the structure of port-Hamiltonian systems. That is, many portHamiltonian techniques (re-design, learning) can be applied on this close-loop systems.

Remark 1 Lemma 3 is easily extend to the case of the following (mechanical) port-Hamiltonian systems with dissipation (frictional effect)

$$
\Sigma_{m r}^{p H}:\left\{\begin{aligned}
{\left[\begin{array}{c}
\dot{q} \\
\dot{p}
\end{array}\right] } & =\left[\begin{array}{cc}
0 & I \\
-I & -R(q, p)
\end{array}\right]\left[\begin{array}{c}
\frac{\partial H_{m}}{\partial q} \\
\frac{\partial H_{m}}{\partial p}
\end{array}\right]+\left[\begin{array}{l}
0 \\
G
\end{array}\right] u \\
y & =G^{T} \frac{\partial H_{m}{ }^{T}}{\partial p}
\end{aligned}\right.
$$

where $R(x)=R(x)^{T}>0$. (See the footnote in Section II.) 


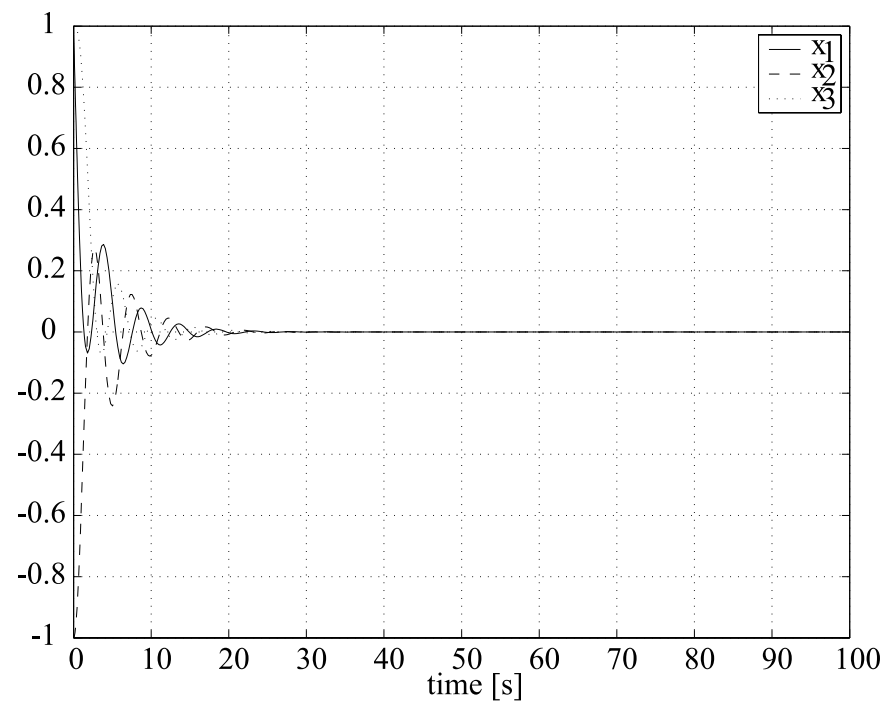

Fig. 3. Time responses of the linear mechanical-spring system

\section{Numerical simulations}

In this section, we confirm the validity of our methods by numerical simulation. Fig. 3 shows the time response of a standard (the simplest) linear mechanical-spiring SISO system with the stabilizer in Theorem 1. This SISO system does not have any damping at all. All states of the closed-loop system $\left(x_{i}, i=1,2,3\right)$ convert to the origin smoothly and the validity of Theorem 1 is confirmed.

Figures $4-5$ show the results by the stabilizer in Lemma 3 . Initial states are $(1,-1,1,1)$ and all parameters are normalized as 1 . Fig. 4 shows the time responses of the state at $D=1 / 2$. Only the state of mechanical systems $(q, p)$ converts to the origin smoothly. This implies that the validity of the partial stabilization methods in Theorem 2 . The settling time is about $20 \mathrm{~s}$. Fig. 5 shows the time responses of the state at $D=1 / 5$. In this case, only the state of mechanical systems $(q, p)$ also converts to the origin smoothly. However the settling time is about $12 \mathrm{~s}$ even though the gain $D$ is lower than $1 / 2$. This implies that the linearized system around the origin has not only poles but also zeros and the gain-tuning guideline Sakai \& Fujimoto (2005) will be useful. (See Sakai \& Fujimoto (2005).) The states converts to the origin. In all, the validity of our methods are confirmed.

\section{Conclusions}

This chapter gives a new modeling and control of hydraulic linear arms. First, we propose two stabilization methods a dynamic asymptotic stabilization method and a partial stabilization method. Second, we give a new model of hydraulic arms using Casimir functions. Third, the proposed two stabilization methods are applied to this new model and a new passivity based control are proposed. Finally, the validity of our methods are confirmed by simulations even though we did not identify the bulk modulus $E$ at all. In future work, not only the friction but also the gravity compensation (which are important in the field applications) will be achieved in the similar approach. 


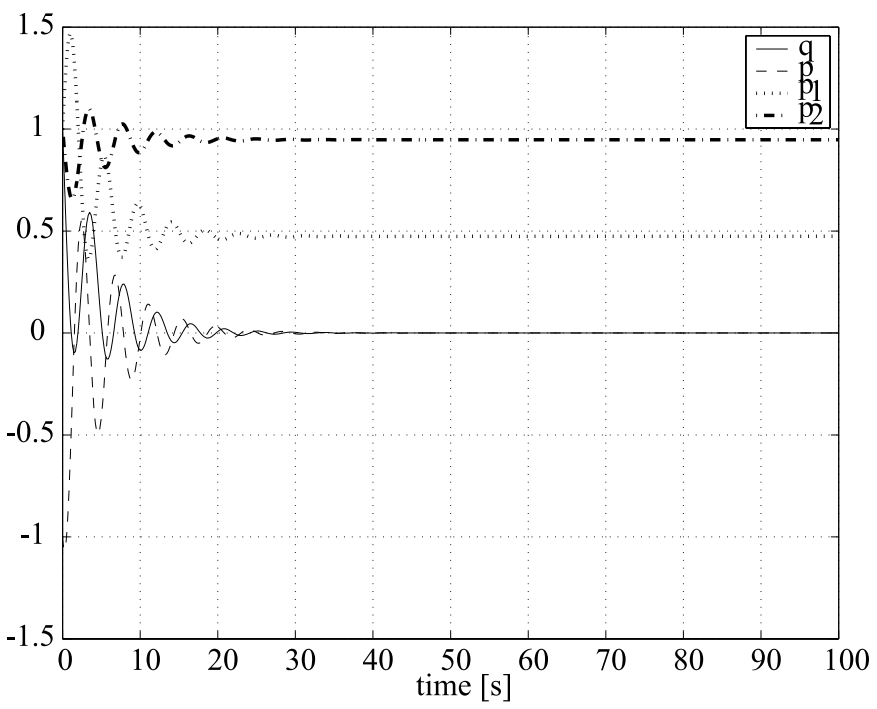

Fig. 4. Time response of all states $(D=1 / 2)$

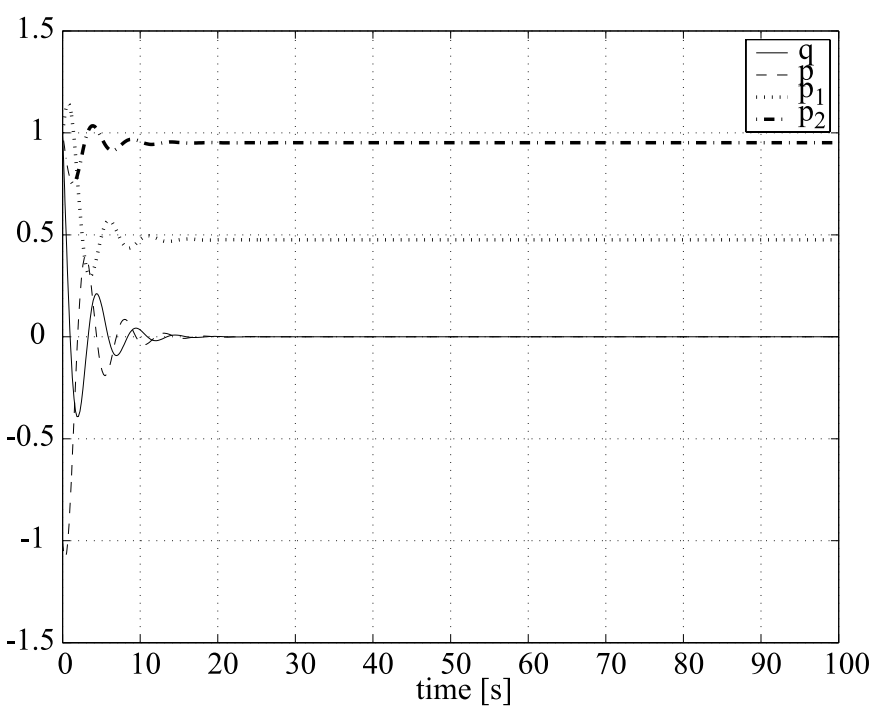

Fig. 5. Time response of all states $(D=1 / 5)$ 


\section{References}

Bonchis, A., Corke, P., D.C.Rye \& Ha, Q. (2001). Variable structure methods in hydraulic servo systems control, Automatica 37(1): 589-595.

Fujimoto, K. \& Sugie, T. (2001). Canonical transformation and stabilization of generalized hamiltonian systems, Systems \& Control Letters 42(3): 217-227.

Gernot, G. \& Schlacher, K. (2005). Energy-based nonlinear control of hydraulically actuated mechanical systems, Proc. of the 44th Conference on Decision and Control, pp. 7520-7525.

Jelali, M. \& Kroll, A. (2002). Hydraulic Servo Systems, Springer.

Khalil, H. K. (1996). Nonlinear Systems, third edn, Macmillan Publishing Company, New York.

Macchelli, A. \& Melchiorri, C. (2005). Control by interconnection of mixed port hamiltonian systems, IEEE Trans. Automatic Control 50(11): 1839-1844.

Maschke, B. M. J. \& van der Schaft, A. J. (1992). Port-controlled Hamiltonian systems: modeling origins and system-theoretic properties, IFAC Symp. Nonlinear Control Systems, pp. 282-288.

Maschke, B. M. J. \& van der Schaft, A. J. (1994). A Hamiltonian approach to stabilization of nonholonomic mechanical systems, Proc. 33rd IEEE Conf. on Decision and Control, pp. 2950-2954.

Mazenc, F. \& Richard, E. (n.d.). Stabilization of hydraulic systems using passive property, Systems and Control Letters 44.

Merrit, H. (1967). Hydraulic Control Systems, John Willey \& Sons.

Ortega, R. \& Garcia-Canseco, E. (2004). Interconection and damping assignment passivitybased control: A survey, European Journal of Control pp. 1-27.

Ramkrishna, P. \& van. der. Schaft, A. (2006). A port-hamiltonian approach to modeling and interconnections of canal systems, Network modeling and control of physical systems, p. WeA08.

Riccardo, M., Roberto, Z. \& Paolo, F. (2006). Dynamic model of an electro-hydraulic three point hitch, Proc. of the 2006 Amecican Control Conference, pp. 1868-1873.

Sakai, S. \& Fujimoto, K. (2005). Dynamic output feedback stabilization of a class of nonholonomic hamiltonian systems, Proc. IFAC World Congress 2005, pp. 1967-1970.

Sakai, S. \& Stramigioli, S. (2007). Port-hamiltonian approaches to motion generations for mechanical systems, Proc. of IEEE Conference on Robotics and Automation, pp. 69-74.

Stramigioli, S., Maschke, B. M. J. \& van der Schaft, A. J. (1998). Passive output feedback and port interconnection, Proc. 4th IFAC Symp. Nonlinear Control Systems, pp. 613-618.

Takegaki, M. \& Arimoto, S. (1981). A new feedback method for dynamic control of manipulators, Trans. ASME, J. Dyn. Syst., Meas., Control 103: 119-125.

van der Schaft, A. J. (2000). L $L_{2}$-Gain and Passivity Techniques in Nonlinear Control, SpringerVerlag, London.

Yao, B., Bu, F., Reedy, J. \& Chiu, G. T.-C. (2000). Adaptive robust motion control of single rod hydraulic actuators: Thorey and experiment, IEEE/ASME Transactions of Mechatoronics 5(1): 79-91.

Zhu, W. \& Piedboeuf, J. (n.d.). Adaptive output force tracking control of hydraulic cylinders with applications to robot manipulators, Transactions of the ASME Dynamic systems, Measurement and Control 127. 


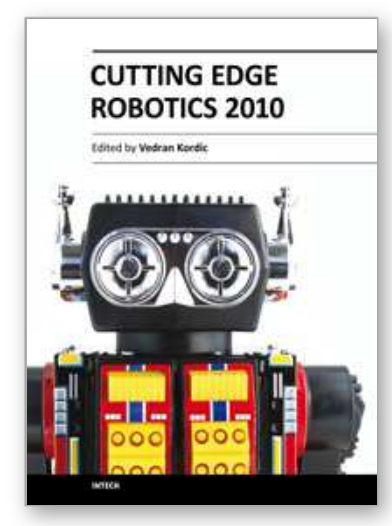

\author{
Cutting Edge Robotics 2010 \\ Edited by Vedran Kordic
}

ISBN 978-953-307-062-9

Hard cover, 440 pages

Publisher InTech

Published online 01, September, 2010

Published in print edition September, 2010

Robotics research, especially mobile robotics is a young field. Its roots include many engineering and scientific disciplines from mechanical, electrical and electronics engineering to computer, cognitive and social sciences. Each of this parent fields is exciting in its own way and has its share in different books. This book is a result of inspirations and contributions from many researchers worldwide. It presents a collection of a wide range of research results in robotics scientific community. We hope you will enjoy reading the book as much as we have enjoyed bringing it together for you.

\title{
How to reference
}

In order to correctly reference this scholarly work, feel free to copy and paste the following:

Satoru Sakai (2010). Passivity Based Control of Hydraulic Linear Arms Using Natural Casimir Functions, Cutting Edge Robotics 2010, Vedran Kordic (Ed.), ISBN: 978-953-307-062-9, InTech, Available from: http://www.intechopen.com/books/cutting-edge-robotics-2010/passivity-based-control-of-hydraulic-linear-armsusing-natural-casimir-functions

\section{INTECH}

open science | open minds

\section{InTech Europe}

University Campus STeP Ri

Slavka Krautzeka 83/A

51000 Rijeka, Croatia

Phone: +385 (51) 770447

Fax: +385 (51) 686166

www.intechopen.com

\section{InTech China}

Unit 405, Office Block, Hotel Equatorial Shanghai

No.65, Yan An Road (West), Shanghai, 200040, China

中国上海市延安西路65号上海国际贵都大饭店办公楼 405 单元

Phone: +86-21-62489820

Fax: +86-21-62489821 
(C) 2010 The Author(s). Licensee IntechOpen. This chapter is distributed under the terms of the Creative Commons Attribution-NonCommercialShareAlike-3.0 License, which permits use, distribution and reproduction for non-commercial purposes, provided the original is properly cited and derivative works building on this content are distributed under the same license. 